\title{
On Equivalence Checking and Logic Synthesis of Circuits with a Common Specification
}

\author{
Eugene Goldberg \\ Cadence Berkeley Labs \\ 1995, University Ave., suite 460, Berkeley,CA, USA, 94704 \\ tel. 1-510-647-2825, email: egold@cadence.com
}

\begin{abstract}
In this paper we develop a theory of equivalence checking (EC) and logic synthesis of circuits with a common specification (CS). We show that two combinational circuits $N_{1}, N_{2}$ have a CS iff they can be partitioned into subcircuits that are connected "in the same way" and are toggle equivalent. This fact allows one to represent a specification of a circuit implicitly as a partitioning into subcircuits. We give an efficient procedure for checking if circuits $N_{1}, N_{2}$ have the same predefined specification. As a "by-product", this procedure performs EC of $N_{1}$ and $N_{2}$. We show how, given a circuit $N_{1}$ with a predefined specification, one can efficiently build a circuit $N_{2}$ satisfying the same specification. We give experimental evidence that EC of $N_{1}, N_{2}$ is hard if their CS is unknown.
\end{abstract}

\section{Categories and Subject Descriptors}

B.6.3 [Logic Design]: Design Aids - automatic synthesis, optimization, verification.

\section{General Terms. Algorithms, Theory, Design,Verification}

Keywords. common specification, scalable equivalence checking, scalable logic synthesis, toggle equivalence.

\section{INTRODUCTION}

In this paper we continue developing the theory of equivalence checking (EC) and logic synthesis of circuits with a common specification (CS) started in [6][4]. A CS $S$ of $N_{1}$ and $N_{2}$ is just a circuit of multi-valued gates (further referred to as blocks) such that $N_{1}$ and $N_{2}$ are different implementations of $S$. Figure 1 gives an example. Circuits $N_{1}$ and $N_{2}$ have a 3-block CS shown on the left. Subcircuits $N_{1}{ }^{\mathrm{i}}, N_{2}{ }^{\mathrm{i}}$ are different implementations of the multi-valued block $G_{\mathrm{i}}$ of $S$. Circuit $N_{\mathrm{m}}{ }^{\mathrm{i}}(m=1,2)$ implements a multi-output Boolean function whose truth table is obtained from that of $G_{\mathrm{i}}$ by replacing values of multi-valued variables with their binary codes. So the difference between $N_{1}{ }^{\mathrm{i}}$ and $N_{2}{ }^{\mathrm{i}}$ is in the choice of binary encodings for the variables of $S$. The size of the largest subcircuit $N_{\mathrm{m}}{ }^{\mathrm{i}}$ is called the granularity of specification $S$ of $N_{\mathrm{m}}$.

Permission to make digital or hard copies of all or part of this work for personal or classroom use is granted without fee provided that copies are not made or distributed for profit or commercial advantage and that copies bear this notice and the full citation on the first page. To copy otherwise, or republish, to post on servers or to redistribute to lists, requires prior specific permission and/or a fee.

GLSVLSI'05, April 17-19, 2005, Chicago, Illinois, USA.

Copyright ACM 1-59593-057-4/05/0004...\$5.00.
There are at least four reasons why a theory of circuits with a CS is of practical importance: a) large circuits are usually composed of a set of meaningful subcircuits; b) the space of implementations of the same specification is very "rich" and so this space most likely contains implementations that are much better than the original one; c) there is an efficient procedure for checking if circuits $N_{1}$ and $N_{2}$ implement the same specification and are functionally equivalent; $d$ ) there is an efficient procedure to build a circuit satisfying a predefined specification.

An example of a large combinational circuit having a high-level structure is a multiplier that is usually specified as a network of smaller subcircuits, adders. A sequential circuit always has a "natural" partitioning into combinational subcircuits implementing next-state functions. (Although the theory we develop in this paper targets synthesis and verification of large combinational circuits with a high-level structure, after some modification, it can be also applied to synthesis and verification of sequential circuits.).

Implementations of the same specification $S$ are different only in the choice of encodings. A $k$-output subcircuit can be considered as an implementation of a function that takes up to $2^{\mathrm{k}}$ values. The number of different $k$-bit encodings of a $2^{\mathrm{k}}$-valued variable is $\left(2^{k}\right)$ ! . This number is very large even for very small values of $k$. So even for a specification $S$ of small granularity the space of implementations is huge.

One of the problems one has to face when building a circuit $N_{2}$ that is another implementation of a specification $S$ of $N_{1}$ is the increasing complexity of EC. Since $N_{1}$ and $N_{2}$ may not have any functionally equivalent internal points, their equivalence cannot be, in general, efficiently checked by the existing algorithms. Fortunately, in [6] it was shown that if a CS $S$ of $N_{1}$ and $N_{2}$ is known, there is an efficient procedure for EC of $N_{1}$ and $N_{2}$. The most advanced version of this procedure is given in [4].

The flaw of the procedure of [4] is in its relying on the assumption that the specifications $S_{1}$ of $N_{1}$ and $S_{2}$ of $N_{2}$ represented by the corresponding partitions are identical i.e. $S_{1}=S_{2}=S$. In this paper, we show that circuits $N_{1}$ and $N_{2}$ have a CS of a specified topology $T$ iff they can be partitioned into subcircuits $N_{1}{ }^{1}, \ldots, N_{1}{ }^{\mathrm{k}}$ and $N_{2}{ }^{1}, \ldots, N_{2}{ }^{\mathrm{k}}$ that are connected as described by $T$ and if $N_{1}{ }^{\mathrm{j}}$ and $N_{2}{ }^{\mathrm{j}}$ are toggle equivalent $j=1, . ., k$. This important result allows one to specify a specification of a circuit implicitly as a partitioning into subcircuits, without representing explicitly the functionality of multi-valued blocks or binary encodings. We modify the procedure of [4] so that now it 
not only checks if $N_{1}$ and $N_{2}$ are functionally equivalent but also tests if specifications of $N_{1}$ and $N_{2}$ are identical.

The notion of toggle equivalence is key in building a circuit $\mathrm{N}_{2}$ that implements the same specification $S$ as $N_{1}$. In this paper we show that if $S$ is specified as a partitioning of $N_{1}$ into subcircuits $N_{1}{ }^{1}, \ldots, N_{1}{ }^{\mathrm{k}}$, to build $N_{2}$ it is sufficient to replace each subcircuit $N_{1}{ }^{\mathrm{i}}$ with its toggle equivalent counterpart $N_{2}{ }^{\mathrm{i}}$.

The paper is structured as follows. Section 2 introduces the notion of toggle equivalence of Boolean functions. In Section 3 we show that circuits $N_{1}$ and $N_{2}$ have a CS iff they can be partitioned into toggle equivalent subcircuits that are connected "in the same way" in $N_{1}$ and $N_{2}$. In Section 4 we give a procedure for checking if a CS of $N_{1}, N_{2}$ is correct. In Section 5 we explain why EC of circuits with unknown CS is hard. In Section 6 we discuss logic synthesis of circuits preserving a predefined specification. In Section 7 we give experimental evidence that EC of circuits with unknown CS is hard. Finally, we draw some conclusions in Section 8.
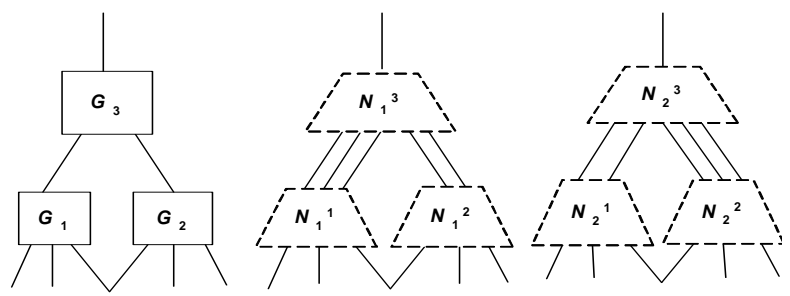

Figure 1. Circuits $N_{1}$ and $N_{2}$ with a common specification of three blocks

\section{TOGGLE EQUIVALENCE}

In this section, we introduce the notion of toggle equivalence. We also show that toggle equivalent Boolean functions can be considered as different implementations of the same multi-valued function.

\subsection{Toggle Equivalence of Boolean Functions with Identical Sets of Variables}

Definition 1. Let $f_{1}:\{0,1\}^{\mathrm{n}} \rightarrow\{0,1\}^{\mathrm{m}}$ and $\boldsymbol{f}_{2}\{0,1\}^{\mathrm{n}} \rightarrow\{0,1\}^{\mathrm{k}}$ be $m$-output and $k$-output Boolean functions of the same set of variables. Functions $f_{1}$ and $f_{2}$ are called toggle equivalent if $\boldsymbol{f}_{\mathbf{1}}(\boldsymbol{x}) \neq \boldsymbol{f}_{\mathbf{1}}\left(\boldsymbol{x}^{\prime}\right) \Leftrightarrow \boldsymbol{f}_{\mathbf{2}}(\boldsymbol{x}) \neq \boldsymbol{f}_{\mathbf{2}}\left(\boldsymbol{x}^{\prime}\right)$. Circuits $N_{1}$ and $N_{2}$ implementing toggle equivalent functions $f_{1}$ and $f_{2}$ are called toggle equivalent circuits.

Informally, toggle equivalence means that for any pair of input vectors $x, x^{\prime}$ for which at least one output of $f_{1}$ "toggles", the same is true for $f_{2}$ and vice versa.

Definition 2. Let $\boldsymbol{f}$ be a multi-output Boolean function of $n$ variables. Denote by $\operatorname{Part}(f)$ the partition of the set $\{0,1\}^{\mathrm{n}}$ into disjoint subsets $B_{1}, \ldots, B_{\mathrm{k}}$ such that $\boldsymbol{f}(\boldsymbol{x})=\boldsymbol{f}\left(\boldsymbol{x}^{\prime}\right)$ iff $\boldsymbol{x}, \boldsymbol{x}^{\prime}$ are in the same subset $B_{\mathrm{i}}$.

Proposition 1. Two Boolean functions $f_{1}$ and $f_{2}$ are toggle equivalent iff $\operatorname{Part}\left(f_{1}\right)=\operatorname{Part}\left(f_{2}\right)$ i.e. iff for each element $B_{\mathrm{i}}$ of the partition $\operatorname{Part}\left(f_{1}\right)$ there is an element $B_{j}^{\prime}$ of the partition $\operatorname{Part}\left(f_{2}\right)$ such that $B_{\mathrm{i}}=B_{\mathrm{j}}^{\prime}$ and vice versa.

Proof. If $f_{1}$ and $f_{2}$ are toggle equivalent, there cannot be a pair of vectors $\boldsymbol{x}, \boldsymbol{x}^{\prime}$ such that $\boldsymbol{x}, \boldsymbol{x}^{\prime}$ are in the same subset of one partition and in different subsets of the other partition. (Because that would mean that one function produces two identical output assignments while the other function toggles.)

Proposition 2. Let $f_{1}$ and $f_{2}$ be toggle equivalent single output Boolean functions. Then $f_{1}=f_{2}$ or $f_{1}=\sim f_{2}$ where ' $\sim$ ' means negation.

Proof. From Proposition 1 it follows that $\operatorname{Part}\left(f_{1}\right)=\operatorname{Part}\left(f_{2}\right)$. Since $f_{1}, f_{2}$ are single output Boolean functions, $\operatorname{Part}\left(f_{1}\right)$ and $\operatorname{Part}\left(f_{2}\right)$ consist of two elements each. So $f_{1}=f_{2}$ or $f_{1}=\sim f_{2}$.

Definition 3. A multi-output Boolean function $\boldsymbol{f}$ of multi-valued variables is called an implementation of a multi-valued function $F$, if the truth table of $\boldsymbol{f}$ can be obtained from that of $F$ in the following two steps

1) Replace the values of multi-valued variables of $F$ with their codes (we assume that different values of a variable are assigned different codes);

2) Fill in the empty rows (if any) of the truth table with arbitrary Boolean vectors.

Proposition 3. Let $f_{1}$ and $f_{2}$ be toggle equivalent. Then $f_{1}$ and $f_{2}$ are two different implementations of the same multi-valued function of Boolean variables.

Proof. According to Proposition 1, $\operatorname{Part}\left(\boldsymbol{f}_{1}\right)=\operatorname{Part}\left(\boldsymbol{f}_{2}\right)$. Let $\operatorname{Part}\left(f_{1}\right), \operatorname{Part}\left(f_{2}\right)$ consist of $k$ elements each. Then $f_{1}$ and $f_{2}$ are implementations of the function $F:\{0,1\}^{\mathrm{n}} \rightarrow\{1, . ., k\}$ where $F(\boldsymbol{x})=m$, iff $\boldsymbol{x} \in B_{\mathrm{m}}$, i.e. iff $\boldsymbol{x}$ is in the $m$-th element of $\operatorname{Part}\left(\boldsymbol{f}_{1}\right)$.

Proposition 3 is of great importance because it shows how one can reencode multi-valued variables implicitly. Suppose, a multioutput circuit $N_{1}$ implements a multi-valued function $F$ and we want to reencode $F$. Synthesizing a circuit $N_{2}$ that is toggle equivalent to $N_{1}$ we obtain a new implementation of $F$. That is we reencode the output multi-valued variable of $F$ not even knowing the binary encodings used to obtain $N_{1}$ and $N_{2}$ from $F$.

\subsection{Toggle Equivalence of Boolean Functions with Different Sets of Variables}

In this subsection, the notion of toggle equivalence is extended to the case of Boolean functions with different sets of variables that are related by constraint functions.

Definition 4. Let $X$ and $Y$ be two disjoint sets of Boolean variables (the number of variables in $X$ and $Y$ may be different). A function $C f(X, Y)$ is called a correlation function if there are subsets $A^{\mathrm{X}} \subseteq\{0,1\}^{|\mathrm{X}|}$ and $A^{\mathrm{Y}} \subseteq\{0,1\}^{|\mathrm{Y}|}$ such that

1) $\left|A^{\mathrm{X}}\right|=\left|A^{\mathrm{Y}}\right|$ and

2) $\quad C f(X, Y)$ specifies a bijective mapping $M: A^{\mathrm{X}} \rightarrow A^{\mathrm{Y}}$. Namely $C f(\boldsymbol{x}, \boldsymbol{y})=1$ iff $\boldsymbol{x} \in A^{\mathrm{X}}$ and $\boldsymbol{y} \in A^{\mathrm{Y}}$ and $\boldsymbol{y}=M(\boldsymbol{x})$.

Remark 1. Informally, $C f(X, Y)$ is a correlation function if it specifies a bijective mapping between a subset $A^{\mathrm{X}}$ of $\{0,1\}^{|\mathrm{X}|}$ and a subset $A^{\mathrm{Y}}$ of $\{0,1\}^{|\mathrm{Y}|}$. So one can view $C f(X, Y)$ as relating two different encodings of an $\left|A^{\mathrm{X}}\right|$ - valued variable.

As Proposition 4 below shows, one can check if a Boolean function $H(X, Y)$ is a correlation one without explicitly finding subsets $A^{\mathrm{X}}$ and $A^{\mathrm{Y}}$.

Proposition 4. Let $X$ and $Y$ be two disjoint sets of Boolean variables. A Boolean function $H(X, Y)$ is a correlation one iff the following two conditions hold: 
1) There do not exist three vectors $x, x^{\prime}, \boldsymbol{y}$ (where $\boldsymbol{x}, \boldsymbol{x}^{\prime}$ are assignments to variables $X$ and $y$ is an assignment to variables $Y$ ) such that $\boldsymbol{x} \neq \boldsymbol{x}^{\prime}$ and $H(\boldsymbol{x}, \boldsymbol{y})=H\left(\boldsymbol{x}^{\prime}, \boldsymbol{y}\right)=1$.

2) There do not exist three vectors $\boldsymbol{x}, \boldsymbol{y}, \boldsymbol{y}^{\prime}$ such that $\boldsymbol{y} \neq \boldsymbol{y}^{\prime}$ and $H(\boldsymbol{x}, \boldsymbol{y})=H\left(\boldsymbol{x}, \boldsymbol{y}^{\prime}\right)=1$.

Proof. Only if part. If $H(X, Y)$ is a correlation function, the fact that conditions 1) and 2) hold, follows from Definition 4.

If part. If conditions 1) and 2) hold then, $H(X, Y)$ specifies a bijective mapping between subsets $A^{\mathrm{X}}$ and $A^{\mathrm{Y}}$ defined in the following way. Subset $A^{\mathrm{X}}$ consists of all the assignments $\boldsymbol{x} \in$ $\{0,1\}^{|\mathrm{X}|}$ such that $H(\boldsymbol{x}, \boldsymbol{y})=1$ for some $\boldsymbol{y} \in\{0,1\}^{|\mathrm{Y}|}$. Subset $A^{\mathrm{Y}}$ consists of all the assignments $\boldsymbol{y} \in\{0,1\}^{|\mathrm{Y}|}$ such that $H(\boldsymbol{x}, \boldsymbol{y})=1$ for some $x \in\{0,1\}^{|\mathrm{X}|}$.

Remark 2. Checking if $H(X, Y)$ is a correlation function reduces to two satisfiability checks. Checking condition 1) of Proposition 4 reduces to testing the satisfiability of the expression $H(X, Y) \wedge$ $H\left(X^{\prime}, Y^{\prime}\right) \wedge N e q\left(X, X^{\prime}\right) \wedge E q\left(Y, Y^{\prime}\right)$. Here $H\left(X^{\prime}, Y^{\prime}\right)$ is a "copy" of $H(X, Y)$ where variables of $X^{\prime}, Y^{\prime}$ are independent of those of $X, Y$. $\operatorname{Neq}\left(\boldsymbol{x}, \boldsymbol{x}^{\prime}\right)$ is equal to 1 iff $\boldsymbol{x} \neq \boldsymbol{x}^{\prime}$. Function $\operatorname{Eq}\left(Y, Y^{\prime}\right)$ is the negation of $\mathrm{Neq}\left(Y, Y^{\prime}\right)$. Checking condition 2) of Proposition 4 reduces to testing the satisfiability of $H(X, Y) \wedge H\left(X^{\prime}, Y^{\prime}\right) \wedge$ $E q\left(X, X^{\prime}\right) \wedge N e q\left(Y, Y^{\prime}\right)$. If both expressions are constant 0 , then $H$ is a correlation function.

Definition 5. Let $f_{1}:\{0,1\}^{\mathrm{n}} \rightarrow\{0,1\}^{\mathrm{m}}$ and $\boldsymbol{f}_{2}:\{0,1\}^{\mathrm{p}} \rightarrow\{0,1\}^{\mathrm{k}}$ be $m$-output and $k$-output Boolean functions and $X$ and $Y$ specify their sets of Boolean variables where $|X|=n$ and $|Y|=p$. Let $D_{\text {inp }}(X, Y)$ be a Boolean function. Functions $f_{1}$ and $f_{2}$ are called toggle equivalent under constraint function $D_{\text {inp }}(X, Y)$ if $\left(f_{1}(x) \neq\right.$ $\left.\boldsymbol{f}_{\mathbf{1}}\left(\boldsymbol{x}^{\prime}\right) \wedge\left(D_{\text {inp }}(\boldsymbol{x}, \boldsymbol{y})=D_{\text {inp }}\left(\boldsymbol{x}^{\prime}, \boldsymbol{y}^{\prime}\right)=1\right)\right) \Rightarrow\left(\boldsymbol{f}_{\mathbf{2}}(\boldsymbol{y}) \neq \boldsymbol{f}_{\mathbf{2}}\left(\boldsymbol{y}^{\prime}\right)\right.$ and vice versa $\left(f_{2}(y) \neq f_{2}\left(y^{\prime}\right) \wedge\left(D_{i n p}(x, y)=D_{i n p}\left(x^{\prime}, y^{\prime}\right)=1\right)\right) \Rightarrow f_{1}(x) \neq f_{1}\left(x^{\prime}\right)$.

Proposition 5. Let $X, Y$ be sets of Boolean variables and $\left\{X_{1}, \ldots, X_{\mathrm{s}}\right\}$ and $\left\{Y_{1}, \ldots, Y_{\mathrm{s}}\right\}$ be partitions of $X$ and $Y$ respectively. Let $C f\left(X_{1}, Y_{1}\right), \ldots, C f\left(X_{\mathrm{s}}, Y_{\mathrm{s}}\right)$ be correlation functions. Let $\boldsymbol{f}_{\mathbf{1}}(X)$ and $\boldsymbol{f}_{\mathbf{2}}(Y)$ be toggle equivalent under the constraint function $D_{\text {inp }}(X, Y)=C f\left(X_{1}, Y_{1}\right) \wedge \ldots \wedge C f\left(X_{\mathrm{s}}, Y_{\mathrm{s}}\right)$. Then $f_{1}$ and $f_{2}$ are implementations of the same multi-valued function of $s$ multivalued variables.

Proof follows from Proposition 3 and Remark 1.

\subsection{Testing Toggle Equivalence}

In this subsection, we show how one can check if multi-output Boolean circuits $N_{1}$ and $N_{2}$ are toggle equivalent. Namely, we show that checking the toggle equivalence of $N_{1}$ and $N_{2}$ reduces to testing if function $D_{\text {out }}\left(N_{1}, N_{2}\right)$ specified by Definition 8 (see below) is a correlation one. This test can be performed by two satisfiability checks as described in Remark 2.

Definition 6. Let $N$ be a Boolean circuit. Denote by $v(N)$ be the set of Boolean variables associated either with the output of a gate or a primary input of $N$. Denote by $\operatorname{Sat}(v(N))$ the Boolean function such that $\operatorname{Sat}(z)=1$ iff the assignment $z$ to variables $v(N)$ is "possible" i.e consistent. For example, if circuit $N$ consists of just one AND gate $y=x_{1} \wedge x_{2}$, then $v(N)=\left\{y, x_{1}, x_{2}\right\}$ and $\operatorname{Sat}(v(N))=$ $\left(\sim x_{1} \vee \sim x_{2} \vee y\right) \wedge\left(x_{1} \vee \sim y\right) \wedge\left(x_{2} \vee \sim y\right)$.

Definition 7. Let $f$ be a Boolean function. We will say that function $f^{*}$ is obtained from $f$ by existentially quantifying away variable $x$ if $f^{*}=f(\ldots, x=0, \ldots) \vee f(\ldots, x=1, \ldots$.$) .$

Definition 8. Let $N_{1}$ and $N_{2}$ be Boolean circuits whose inputs are specified by set of variables $X$ and $Y$ respectively. Let
$D_{\text {inp }}(X, Y)$ be a Boolean function. Denote by $D_{\text {out }}\left(N_{1}, N_{2}\right)$ the Boolean function obtained from the Boolean function $H$, where $H=\operatorname{Sat}\left(v\left(N_{1}\right)\right) \wedge \operatorname{Sat}\left(v\left(N_{2}\right)\right) \wedge D_{\text {inp }}(X, Y)$, by existentially quantifying away all the variables of $H$ but the output variables of $N_{1}$ and $N_{2}$.

Proposition 6. Let $N_{1}$ and $N_{2}$ be Boolean circuits with input variables specified by sets $X, Y$ respectively. Let $D_{\text {inp }}(X, Y)$ be a Boolean function relating $X$ and $Y$. Let $D_{\text {inp }}(X, Y)$ be a correlation function. Then $N_{1}$ and $N_{2}$ are toggle equivalent under constraint function $D_{\text {inp }}(X, Y)$ iff the function $D_{\text {out }}\left(N_{1}, N_{2}\right)$ specified in Definition 8 is also a correlation function.

Proof. Only If part. Let $N_{1}$ and $N_{2}$ be toggle equivalent. Then $D_{\text {out }}\left(N_{1}, N_{2}\right)$ satisfies either condition of Proposition 4 and hence it is a correlation function. For example, there cannot exist Boolean vectors $\boldsymbol{z}, \boldsymbol{z}^{\prime}$ and $\boldsymbol{h}$ (where $\boldsymbol{z} \neq \boldsymbol{z}^{\prime}$ and $\boldsymbol{z}, \boldsymbol{z}^{\prime}$ are output assignments of $N_{1}$ and $\boldsymbol{h}$ is an output assignment of $N_{2}$ ) such that $D_{\text {out }}(z, \boldsymbol{h})=D_{\text {out }}\left(z^{\prime}, \boldsymbol{h}\right)=1$. Indeed, it would mean that there exist pairs of vectors $\boldsymbol{x}, \boldsymbol{y}$ and $\boldsymbol{x}^{\prime}, \boldsymbol{y}^{\prime}$ such that a) $z=N_{1}(\boldsymbol{x}), \boldsymbol{z}^{\prime}=N_{2}\left(\boldsymbol{x}^{\prime}\right)$ and $\left.\boldsymbol{h}=N_{2}(\boldsymbol{y})=N_{2}\left(\boldsymbol{y}^{\prime}\right) ; \mathrm{b}\right) \quad D_{\text {inp }}(\boldsymbol{x}, \boldsymbol{y})=1$ and $D_{\text {inp }}\left(\boldsymbol{x}^{\prime}, \boldsymbol{y}^{\prime}\right)=1 ;$ c) $\boldsymbol{x} \neq \boldsymbol{x}^{\prime}$ and $\boldsymbol{y} \neq \boldsymbol{y}^{\prime}$; d) $N_{1}(\boldsymbol{x}) \neq N_{1}\left(\boldsymbol{x}^{\prime}\right)$ while $N_{2}(\boldsymbol{y})=N_{2}\left(\boldsymbol{y}^{\prime}\right)$. But this is impossible because $N_{1}$ and $N_{2}$ are toggle equivalent.

If part can be proven in a similar manner.

\section{COMMON SPECIFICATION AND TOGGLE EQUIVALENCE}

In this section, we show that the existence of a CS of single output combinational circuits $N_{1}$ and $N_{2}$ means that $N_{1}, N_{2}$ can be partitioned into toggle equivalent subcircuits that are connected in $N_{1}$ and $N_{2}$ "in the same way". The main result of this section is formulated in Proposition 7.
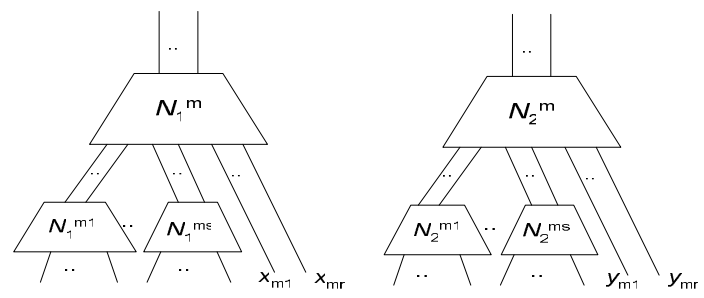

Figure 2. Illustration to Definition 12

Definition 9. Let $N=(V, E)$ be a DAG representing a Boolean circuit (here $V, E$ are sets of nodes and edges of $N$ respectively.) A subgraph $N^{*}=\left(V^{*}, E^{*}\right)$ of $N$ is called a subcircuit if the following two conditions hold:

a) if $g_{1}, g_{2}$ are in $V^{*}$ and there is a path from $g_{1}$ to $g_{2}$ in $N$, then all the nodes of $N$ that on that path are in $V^{*}$;

b) if $g_{1}, g_{2}$ of $V^{*}$ are connected by an edge in $N$, then they are also connected by an edge in $N^{*}$.

Definition 10. Let a Boolean circuit $N$ be partitioned into $k$ subcircuits $N^{1}, \ldots, N^{\mathrm{k}}$. Let $T$ be a directed graph of $k$ nodes such that nodes $G_{\mathrm{i}}$ and $G_{\mathrm{j}}$ of $T$ are connected by a directed edge (from $G_{\mathrm{i}}$ to $G_{\mathrm{j}}$ ) iff an output of $N^{\mathrm{i}}$ is connected to an input of $N^{\mathrm{k}}$ in $N$. $T$ is called the communication specification corresponding to the partition $N^{1}, \ldots, N^{\mathrm{k}}$. The partition $N^{1}, \ldots, N^{\mathrm{k}}$ is called topological if $T$ is a DAG (i.e. if $T$ does not contain cycles)

Definition 11. Let $T$ be the communication specification of circuit $N$ with respect to a topological partition $N^{1}, \ldots, N^{\mathrm{k}}$. Let $G_{\mathrm{i}}$ 
be the node of $T$ corresponding to subcircuit $N^{\mathrm{i}}$. The length of the longest path from an input of $T$ to $G_{\mathrm{i}}$ is called the level of $G_{\mathrm{i}}$ and $N^{\mathrm{i}}$ (denoted by level $\left(G_{\mathrm{i}}\right)$ and $\operatorname{level}\left(N^{\mathrm{i}}\right)$ respectively).
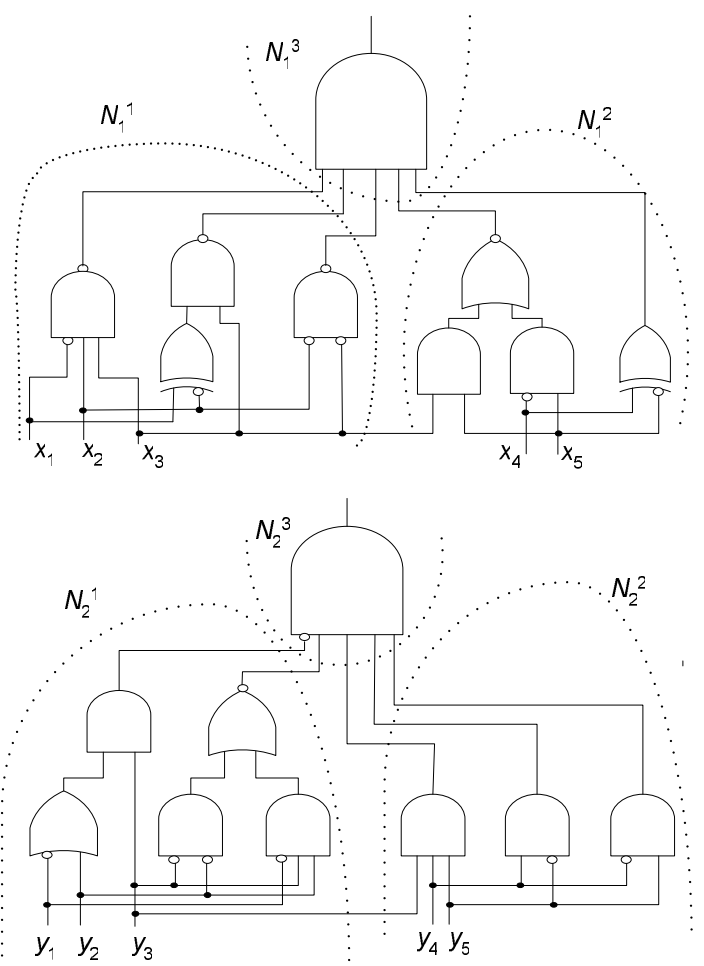

Figure 3. Example of circuits $N_{1}$ and $N_{2}$ with a CS

Definition 12. Let $N_{1}{ }^{1}, \ldots, N_{1}{ }^{\mathrm{k}}$ and $N_{2}{ }^{1}, \ldots, N_{2}{ }^{\mathrm{k}}$ be topological partitions of single output Boolean circuits $N_{1}, N_{2}$. Let communication specifications of $N_{1}$ and $N_{2}$ with respect to partitions $N_{1}{ }^{1}, \ldots, N_{1}{ }^{\mathrm{k}}$ and $N_{2}{ }^{1}, \ldots, N_{2}{ }^{\mathrm{k}}$ be identical. Denote by $D_{\text {out }}\left(N_{1}{ }^{m}, N_{2}{ }^{m}\right), m=1, \ldots, k$ functions computed by induction in topological levels. Namely, we first compute functions $D_{\text {out }}$ for the subcircuits of level 1, then for those of level 2 and so on. Function $D_{\text {out }}\left(N_{1}{ }^{\mathrm{m}}, N_{2}{ }^{\mathrm{m}}\right)$ is obtained from function $H=\operatorname{Sat}\left(v\left(N_{1}{ }^{\mathrm{m}}\right)\right)$ $\wedge \operatorname{Sat}\left(v\left(N_{2}{ }^{\mathrm{m}}\right)\right) \wedge D_{\text {inp }}\left(N_{1}{ }^{\mathrm{m}}, N_{2}{ }^{\mathrm{m}}\right)$ by existentially quantifying away all the variables except the output variables of $N_{1}{ }^{\mathrm{m}}, N_{2}{ }^{\mathrm{m}}$. The function $D_{\text {inp }}\left(N_{1}{ }^{\mathrm{m}}, N_{2}{ }^{\mathrm{m}}\right)$ is equal to $D_{\text {out }}\left(N_{1}{ }^{\mathrm{ml}}, N_{2}{ }^{\mathrm{ml}}\right) \wedge \ldots \wedge$ $D_{\text {out }}\left(N_{1}{ }^{\mathrm{ms}}, N_{2}{ }^{\mathrm{ms}}\right) \wedge E q\left(x_{\mathrm{m} 1}, y_{\mathrm{m} 1}\right) \wedge \ldots \wedge E q\left(x_{\mathrm{mr}}, y_{\mathrm{mr}}\right)$. Here $N_{1}{ }^{\mathrm{m} 1}$, ,.., $N_{1}{ }^{\mathrm{ms}}, N_{2}{ }^{\mathrm{ml}}, \ldots, N_{2}{ }^{\mathrm{ms}}$ are the $s$ subcircuits (if any) whose outputs are connected to inputs of $N_{1}{ }^{\mathrm{m}}, N_{2}{ }^{\mathrm{m}}$ respectively. (See illustration in Figure 2.) Variables $x_{\mathrm{m} 1}, \ldots, x_{\mathrm{mr}}, y_{\mathrm{m} 1}, \ldots, y_{\mathrm{mr}}$ are the $r$ primary input variables of $N_{1}$ and $N_{2}$ (if any) that feed $N_{1}{ }^{\mathrm{m}}$ and $N_{2}{ }^{\mathrm{m}}$ respectively. Function $E q\left(x_{\mathrm{mt}}, y_{\mathrm{mt}}\right), 1 \leq t \leq r$ is equal to 1 iff $x_{\mathrm{mt}}$ is equal to $y_{\mathrm{mt}}$.

Proposition 7. Let $N_{1}, N_{2}$ be two functionally equivalent single output circuits. Let $T$ be a DAG of $k$ nodes. Circuits $N_{1}$ and $N_{2}$ are implementations of a specification $S$ whose topology is given by $T$ iff there is a partitioning $\operatorname{Spec}\left(N_{1}\right)=\left\{N_{1}{ }^{1}, \ldots, N_{1}{ }^{\mathrm{k}}\right\}$ of $N_{1}$ and a partitioning $\operatorname{Spec}\left(N_{2}\right)=\left\{N_{2}{ }^{1}, \ldots, N_{2}{ }^{\mathrm{k}}\right\}$ of $N_{2}$ into $k$ subcircuits such that

a) Communication specifications $T_{1}, T_{2}$ of $N_{1}$ and $N_{2}$ with respect to partitionings $\operatorname{Spec}\left(N_{1}\right), \operatorname{Spec}\left(N_{2}\right)$ are equal to $T$; b) Each pair of circuits $N_{1}{ }^{\mathrm{m}}, N_{2}{ }^{\mathrm{m}}$ is toggle equivalent under constraint function $D_{\text {inp }}\left(N_{1}{ }^{\mathrm{m}}, N_{2}{ }^{\mathrm{m}}\right)$ specified by Definition 12 .

Sketch of the proof. If part. It is proven by induction (in levels) using Proposition 5 and Proposition 6. Only if part is proven by induction using the fact that two Boolean functions implementing the same multi-valued function are toggle equivalent.

An example of circuits with a CS of three blocks is shown in Figure 3. Circuit $N_{1}$ (at the top) and $N_{2}$ (at the bottom) have the same communication specification (shown in Figure 1 on the left side). Subcircuits $N_{1}{ }^{1}, N_{1}{ }^{2}$ (outlined by the dotted line) are toggle equivalent to subcircuits $N_{2}{ }^{1}, N_{2}{ }^{2}$ respectively in terms of their inputs related by the constraint functions $D_{\text {inp }}\left(N_{1}{ }^{1}, N_{2}{ }^{1}\right)=E q\left(x_{1}, y_{1}\right)$ $\wedge E q\left(x_{2}, y_{2}\right) \wedge E q\left(x_{3}, y_{3}\right)$ and $D_{\text {inp }}\left(N_{1}{ }^{2}, N_{2}{ }^{2}\right)=E q\left(x_{3}, y_{3}\right) \wedge$ $E q\left(x_{4}, y_{4}\right) \wedge E q\left(x_{5}, y_{5}\right)$ respectively. Subcircuits $N_{1}{ }^{3}$ and $N_{2}{ }^{3}$ are toggle equivalent in terms of their local inputs related by the constraint function $D_{\text {inp }}\left(N_{1}{ }^{3}, N_{2}{ }^{3}\right)=D_{\text {out }}\left(N_{1}{ }^{1}, N_{2}{ }^{1}\right) \wedge D_{\text {out }}\left(N_{1}{ }^{2}, N_{2}{ }^{2}\right)$. The functions $D_{\text {out }}\left(N_{1}{ }^{1}, N_{2}{ }^{1}\right), \quad D_{\text {out }}\left(N_{1}{ }^{2}, N_{2}^{2}\right)$ are obtained as described in Definition 12.

\section{COMMON SPECIFICATION VERIFICATION PROCEDURE}

In this section, we describe a procedure for checking if a predefined CS of circuits $N_{1}, N_{2}$ is correct. We will refer to this procedure as Common Specification Verification $(C S V)$. As a "by-product", our CSV procedure checks if $N_{1}, N_{2}$ are functionally equivalent.

The EC procedure for circuits $N_{1}, N_{2}$ with a CS introduced in [6] and modified in [4] essentially uses an implicit representation of this CS as partitions of $N_{1}$ and $N_{2}$. Proposition 7 allows one to modify this procedure so that in addition to $\mathrm{EC}$ it also checks the correctness of the CS. The pseudocode of the CSV procedure is shown in Figure 4.

The procedure topol_partition checks if $\operatorname{Spec}\left(N_{1}\right)$ and $\operatorname{Spec}\left(N_{1}\right)$ are topological partitions (see Definition 10). The procedure equiv_commun_specs checks if communication specifications $T_{1}$ of $N_{1}$ with respect to $\operatorname{Spec}\left(N_{1}\right)$ and $T_{2}$ of $N_{2}$ with respect to $\operatorname{Spec}\left(N_{2}\right)$ are identical.

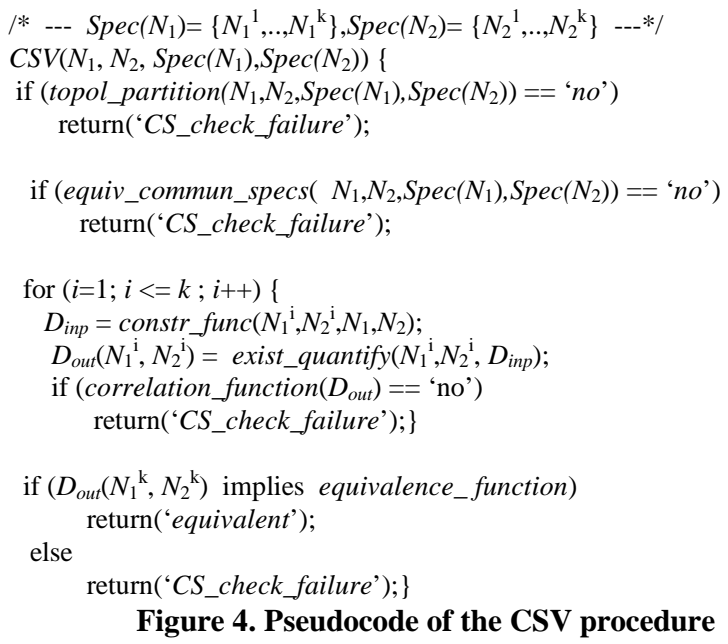

Figure 4. Pseudocode of the CSV procedure 
In the main loop, functions $D_{\text {out }}\left(N_{1}{ }^{\mathrm{i}}, N_{2}\right)$ are computed in topological order as described in Definition 12. Before computing $D_{\text {out }}\left(N_{1}{ }^{\mathrm{i}}, N_{2}{ }^{\mathrm{i}}\right)$ the procedure constr_func forms the expression $D_{\text {inp }}$ (see Definition 12). The function exist_quantify existentially quantifies away from the function $H=\operatorname{Sat}\left(v\left(N_{1}{ }^{\mathrm{m}}\right)\right) \wedge \operatorname{Sat}\left(v\left(N_{2}{ }^{\mathrm{m}}\right)\right) \wedge$ $D_{\text {inp }}$ all the variables except the output variables of $N_{1}{ }^{\mathrm{i}}$ and $N_{2}{ }^{\mathrm{i}}$. Then the correlation function procedure checks if the result of quantification $D_{\text {out }}$ is a correlation function. The check is performed as described in Remark 2.

Finally, the CSV procedure checks if the correlation function of subcircuits $N_{1}{ }^{\mathrm{k}}$ and $N_{2}{ }^{\mathrm{k}}$ (whose primary outputs are primary outputs of $N_{1}$ and $N_{2}$ ) implies the equivalence function $E q(y, z)$. (Here $y, z$ are Boolean variables associated with the outputs of $N_{1}$ and $N_{2}$ respectively). If so, then $N_{1}$ and $N_{2}$ are declared equivalent. Otherwise, the CSV procedure returns the ' $C S \_$check_failure' answer. This answer is also returned if any of the checks performed by topol_partition, equiv_commun_specs and correlation_function fails.

Definition 13. Let $N_{1}, N_{2}$ be two functionally equivalent circuits with a CS $S$ represented by partitions $\operatorname{Spec}\left(N_{1}\right)=\left\{N_{1}{ }^{1}, \ldots, N_{1}{ }^{\mathrm{k}}\right\}$, and $\operatorname{Spec}\left(N_{2}\right)=\left\{N_{2}{ }^{1}, \ldots, N_{2}{ }^{\mathrm{k}}\right\}$. The granularity of $S$ is the size (i.e. the number of gates) of the largest subcircuit $N_{\mathrm{i}}^{\mathrm{j}}, i=1,2, j=1, . ., k$.

The CSV procedure is exponential in the granularity $p$ of $S$ and linear in the number of blocks of $S$ i.e. in the number of subcircuits in $\operatorname{Spec}\left(N_{1}\right), \operatorname{Spec}\left(N_{2}\right)$. The exponentiality in $p$ is due to procedures exist_quantify and correlation_function. The reason why the CSV procedure is exponential only in $p$ and not in the circuit size is that the two exponential procedures above are applied only to subcircuits $N_{1}{ }^{\mathrm{i}}, N_{2}{ }^{\mathrm{i}}$ whose size is bounded by $p$. Suppose that the value of $p$ is bounded by a constant (i.e. circuits $N_{1}, N_{2}$ can be of arbitrary size but the granularity of their CS is bounded). Then the CSV procedure proves the equivalence of specifications $\operatorname{Spec}\left(N_{1}\right)$ and $\operatorname{Spec}\left(N_{2}\right)$ (and hence functional equivalence of $N_{1}$ and $N_{2}$ ) in linear time in the circuit size.

\section{EQUIVALENCE CHECKING WITH UNKNOWN SPECIFICATION}

Note that the efficiency of our CSV procedure is due to the fact that a CS specification of $N_{1}$ and $N_{2}$ (represented by $\operatorname{Spec}\left(N_{1}\right)$ and $\operatorname{Spec}\left(N_{2}\right)$ ) is known. A natural question to ask is as follows. Suppose circuits $N_{1}, N_{2}$ have a CS specification $S$ of small granularity $p$. Is there an efficient procedure for EC of $N_{1}, N_{2}$ if $S$ is unknown (i.e we do not know the partitions $\operatorname{Spec}\left(N_{1}\right)$ and $\operatorname{Spec}\left(N_{2}\right)$ representing $\left.S\right)$ ? In [6] it was conjectured that in that case EC of $N_{1}, N_{2}$ is hard for any deterministic algorithm. The new (and equivalent) definition of CS given in this paper allows one to get a better perspective on the problems one has to solve when checking $N_{1}, N_{2}$ for equivalence.

One way to do the job is to find $\operatorname{Spec}\left(N_{1}\right)$ and $\operatorname{Spec}\left(N_{2}\right)$ and apply the CSV procedure. This approach is very similar to what the existing EC procedures exploiting structural similarity of $N_{1}, N_{2}$ do ([1][2][3][7][8]). Namely, they try to find pairs of functionally equivalent points of $N_{1}, N_{2}$ and use them as cut points. Then new points of $N_{1}, N_{2}$ that are functionally equivalent in terms of cut points are looked for. The idea is that checking functional equivalence of internal points of $N_{1}, N_{2}$ in terms of cut points is much easier than in terms of primary inputs. This approach faces the following two problems. The first problem is to find new potential cut points (i.e. to find points of $N_{1}, N_{2}$ that are functionally equivalent). The second problem is to decide whether two functionally equivalent internal points can be used as cut points. Making a wrong decision here leads to the appearance of so called "false negatives".

One can view the "cut advancement" approach above as search for a CS of $N_{1}, N_{2}$ of a special type where every subcircuit of $\operatorname{Spec}\left(N_{1}\right)$ and $\operatorname{Spec}\left(N_{2}\right)$ has exactly one output. However, if one try to extend this approach to CSs of the general type (where subcircuits of $\operatorname{Spec}\left(N_{1}\right)$ and $\operatorname{Spec}\left(N_{2}\right)$ may have many outputs), the two problems mentioned above become virtually unsolvable. In the case of multi-output subcircuits, functional equivalence is replaced with toggle equivalence. Let the granularity $p$ of a CS of $N_{1}, N_{2}$ be equal to 10 . (So the subcircuits of $\operatorname{Spec}\left(N_{1}\right)$ and $\operatorname{Spec}\left(N_{2}\right)$ may have up to 10 outputs.) Then the number of candidate subcircuits in $N_{1}$ and $N_{2}$ is proportional to $\left|N_{1}\right|^{10}$ and $\left|N_{2}\right|^{10}$ respectively where $\left|N_{\mathrm{j}}\right|$ is the size of $N_{\mathrm{j}}$. The number of potential pairs of subcircuits to examine is proportional to $\left|N_{1}\right|^{10} *\left|N_{2}\right|^{10}$. But even if one finds subcircuits $N_{1}{ }^{\mathrm{i}}, N_{2}{ }^{\mathrm{i}}$ of size less or equal to 10 that are toggle equivalent, one still needs to decide if the outputs of $N_{1}{ }_{1}{ }^{1} N_{2}{ }^{\mathrm{i}}$ can be used as cut points. That is one needs to decide whether $N_{1}{ }^{\mathrm{i}}, N_{2}{ }^{\mathrm{i}}$ are toggle equivalent "by chance" or they are a part of a CS. Since the number of candidates is huge, making a mistake becomes unavoidable.

\section{ON LOGIC SYNTHESIS PRESERVING PREDEFINED SPECIFICATION}

In this section, we describe a procedure that, given a circuit $N_{1}$ with a known specification, builds another circuit $\mathrm{N}_{2}$ implementing the same specification as $N_{1}$.

Let $N_{1}$ be a Boolean circuit that needs to be optimized. Let $S$ be a specification of $N_{1}$ represented as $\operatorname{Spec}\left(N_{1}\right)=\left\{N_{1}{ }^{1}, \ldots, N_{1}{ }^{\mathrm{k}}\right\}$. Figure 5 shows pseudocode of a procedure for generating a circuit $N_{2}$ that implements the same specification as circuit $N_{1}$. We will refer to it as Specification Preserving $(\boldsymbol{S P})$ procedure. We assume that subcircuits $N_{1}{ }^{1}, \ldots, N_{1}{ }^{\mathrm{k}}$ are numbered in topological order i.e. for every pair $i, j$ such that $i<j \Rightarrow \operatorname{level}\left(N_{1}{ }^{\mathrm{i}}\right) \leq \operatorname{level}\left(N_{1}{ }^{\mathrm{j}}\right)$.

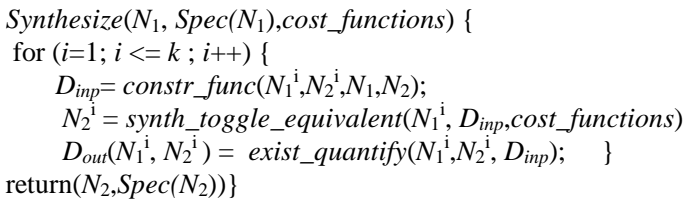

Figure 5. Pseudo code of the SP procedure

The idea of the SP procedure is to replace subcircuits $\left\{N_{1}{ }^{1}, \ldots, N_{1}{ }^{\mathrm{k}}\right\}$ with toggle equivalent subcircuits $\left\{N_{2}{ }^{1}, \ldots, N_{2}{ }^{\mathrm{k}}\right\}$ in topological order moving from inputs to outputs. The SP procedure returns circuit $N_{2}$ implementing the same specification as $N_{1}$. Circuit $N_{2}{ }^{\mathrm{i}}$ toggle equivalent to $N_{1}{ }^{\mathrm{i}}$ is built by the synth_toggle_equivalent procedure. (Describing an implementation of this procedure is beyond the scope of this paper. Some encouraging preliminary results are reported in [5]. ) After $N_{2}{ }^{i}$ is synthesized we compute the correlation function $D_{\text {out }}\left(N_{1}{ }^{\mathrm{i}}, N_{2}{ }^{\mathrm{i}}\right)$ using previously computed functions $D_{\text {out }}$ exactly as it is done by the CSV procedure. (Note that since $N_{1}{ }^{\mathrm{i}}, N_{2}{ }^{\mathrm{i}}$ are toggle equivalent "by construction", $D_{\text {out }}\left(N_{1}{ }^{\mathrm{i}}, N_{2}{ }^{\mathrm{i}}\right)$ is a correlation function.) 
The importance of the SP procedure is twofold. First, the complexity of the SP procedure is the same as that of the CSV procedure. Namely, it is exponential in the granularity $p$ of the CS of $N_{1}, N_{2}$ represented by $\operatorname{Spec}\left(N_{1}\right), \operatorname{Spec}\left(N_{2}\right)$ and linear in the number of subcircuits in $\operatorname{Spec}\left(N_{1}\right)$ and $\operatorname{Spec}\left(N_{2}\right)$. (Here we make a realistic assumption that synth_toggle_equivalent is "only" exponential in $p$ ). This means that if $p$ is fixed, the SP procedure is linear in circuit size and hence it is scalable.

Second, the SP procedure allows one to make a nice trade-off between optimization quality and efficiency. Note that the search space explored by the SP procedure is limited to the implementations of the specification of $N_{1}$ represented by $\operatorname{Spec}\left(N_{1}\right)$. The smaller the granularity $p$ of the specification $S$ of $N_{1}$ is, the smaller the search space is and the greater the efficiency of the SP procedure is. So, if no good alternative implementation $\mathrm{N}_{2}$ is found for the current specification of $N_{1}$, one can merge some adjacent subcircuits of $\operatorname{Spec}\left(N_{1}\right)$ to get a specification of larger granularity. This way the search space becomes larger at the expense of performance degradation of the SP procedure.

\section{EXPERIMENTAL RESULTS}

In this section, we give some experimental results. In the experiments we compared the performance of two EC algorithms: our CSV procedure shown in Figure 4 and an Industrial Equivalence Checker (referred to as IEC) of very high quality. Both algorithms were run on a $3.06 \mathrm{GHz}$ Xeon PC.

Table 1. EC of circuits obtained from 4-valued specifications

\begin{tabular}{|l|l|l|l|l|}
\hline Name & $\begin{array}{l}\# \text { blocks in } \\
\text { CS }\end{array}$ & $\begin{array}{l}\text { CSV } \\
(\mathrm{sec} .)\end{array}$ & $\begin{array}{l}\text { IEC } \\
(\mathrm{sec} .)\end{array}$ & $\begin{array}{l}\text { Ratio } \\
\text { (IEC / CSV) }\end{array}$ \\
\hline $\operatorname{des} 1$ & 705 & 0.4 & 3 & $\mathbf{7}$ \\
\hline $\operatorname{des} 2$ & 2,562 & 2 & 14 & $\mathbf{7}$ \\
\hline $\operatorname{des} 3$ & 3,519 & 3 & 281 & $\mathbf{9 4}$ \\
\hline $\operatorname{des} 4$ & 8,628 & 14 & 308 & $\mathbf{2 2}$ \\
\hline $\operatorname{des} 5$ & 9,027 & 16 & 543 & $\mathbf{3 4}$ \\
\hline $\operatorname{des} 6$ & 10,572 & 20 & 534 & $\mathbf{2 7}$ \\
\hline
\end{tabular}

Table 2. EC of circuits obtained from 8-valued specifications

\begin{tabular}{|l|l|l|l|l|}
\hline Name & $\begin{array}{l}\text { \# blocks in } \\
\text { CS }\end{array}$ & $\begin{array}{l}\text { CSV } \\
(\text { sec. })\end{array}$ & $\begin{array}{l}\text { IEC } \\
(\text { sec. })\end{array}$ & $\begin{array}{l}\text { Ratio } \\
\text { (IEC / CSV) }\end{array}$ \\
\hline des1 & 705 & 5 & $16,948^{*}$ & $>\mathbf{3 , 3 9 0}$ \\
\hline $\operatorname{des} 2$ & 2,562 & 19 & $24,638^{*}$ & $>\mathbf{1 , 2 9 7}$ \\
\hline $\operatorname{des} 3$ & 3,519 & 29 & $>36,000$ & $>\mathbf{1 , 2 4 1}$ \\
\hline $\operatorname{des} 4$ & 8,628 & 107 & 26,758 & $\mathbf{2 5 0}$ \\
\hline $\operatorname{des} 5$ & 9,027 & 111 & $>36,000$ & $\mathbf{> 3 2 4}$ \\
\hline $\operatorname{des} 6$ & 10,572 & 141 & 27,391 & $\mathbf{1 9 4}$ \\
\hline
\end{tabular}

In the experiments we checked for equivalence circuits obtained from a specification given as a combinational circuit of multivalued blocks. The number of values taken by the variables of a block was parameterized. Circuits $N_{1}, N_{2}$ to be checked for equivalence were obtained from a specification using two sets of random encodings of the minimum (logarithmic) length.
The goal of experiments was twofold. First we wanted to show that EC of circuits with a CS $S$ of even small granularity is hard if $S$ is unknown. Second, we wanted to demonstrate that this weakness of current EC algorithms hinders the development of more powerful synthesis procedures. (Even though we obtained circuits $N_{1}, N_{2}$ by explicitly encoding multi-valued variables of a specification, circuit $N_{2}$ could have been obtained from $N_{1}$ by the synthesis procedure described in Section 6.)

In Table 1 we consider specifications with blocks of 4 -valued variables. Second column gives the number of blocks for each design. Third and fourth columns give runtimes for CSV and IEC. The last column gives the ratio of runtimes. In Table 2 we consider the same specifications (i.e. the topology of corresponding specifications was the same) of 8 -valued blocks. Hence the granularity of CSs of binary circuits obtained by encoding multi-valued variables was slightly larger than for binary circuits of Table 1. Runtimes of IEC marked with '*' correspond to the cases where IEC aborted without completion (due to exhausting some internal resource).

For the circuits from both tables CSV was faster than IEC. However the gap between the performance of CSV and IEC increased dramatically as the granularity of specifications had grown. IEC was able to complete all the instances of Table 1 in a reasonable time. On the other hand, it completed only 2 equivalence checks for the circuits of Table 2 and took dramatically more time.

\section{CONCLUSIONS}

In this paper, we show that two combinational circuits $N_{1}, N_{2}$ have a CS $S$ iff they can be partitioned into toggle equivalent subcircuits. We give an efficient procedure for verifying a CS of $N_{1}, N_{2}$ that also performs EC of $N_{1}, N_{2}$. We show how one can build a combinational circuit that preserves a predefined specification. Finally, we give experimental evidence that EC of circuits with unknown CS is hard.

\section{REFERENCES}

[1] Berman,C.L., and Trevillyan,L.H. Functional comparison of logic designs for VLSI circuits. ICCAD-89, pp.456-459.

[2] Brand,D. Verification of large synthesized designs. ICCAD93,pp.534-537.

[3] Burch, J.R., and Singhal,V. Tight integration of combinational verification methods. ICCAD-98, pp.570-576.

[4] Goldberg, E. Equivalence Checking of Dissimilar Circuits II. Technical report. CDNL-TR-2004-0830, August 2004, available at http://eigold.tripod.com/papers.html .

[5] Goldberg, E. Equivalence checking and logic synthesis of circuits with a common specification. Technical report. CDNL-TR-2004-1220, August 2004.

[6] Goldberg, E., and Novikov, Y. Equivalence Checking of Dissimilar Circuits, IWLS-2003. May 28-30, USA. Available at http://eigold.tripod.com/papers/dissim-iwls.zip .

[7] van Eijk, C. and Janssen, G. Exploiting structural similarities in a BDD-based verification method. Proceedings of $2^{\text {nd }}$ International Conference on Theorem Provers in Circuit Design, pp.110-125,1995.

[8] Kuehlmann, A. and Krohm, F. Equivalence checking using cuts and heaps, DAC-98, pp.263-268. 